\title{
Spherical Tiling with GeoGebra *
}

\author{
New Results, Challenges and Open Problems
}

\section{Ana Breda and José Dos Santos}

The theory of spherical tilings is an interesting and fruitful field, attracting, among other researchers, mathematicians. It is a transverse topic crossing several mathematical areas such as geometry, algebra, topology and number theory, but it is also an object of interest for other scientific fields such as chemistry, physics, art and architecture. Here, we make use of GeoGebra to establish some results, describing a class of monohedral spherical tilings and inferring some conjectures. This will highlight how the use of this software has been crucial for the construction of new knowledge in mathematics with applications in different areas of engineering.

\section{Introduction}

The efficiency of arrangements and patterns (packing, covering and tiling) have been the object of study of many generations of mathematicians. In fact, Euclid and Archimedes were deeply interested in this type of problem.

The side by side spherical tilings by congruent polygons (monohedral tilings) have been extensively studied, being the triangular case completely classified, $[1,2]$.

There are many tools to work with spherical geometry interactively such as Sphaerica [3], Spherical Easel [4], and Povray [5]. However, for our purposes, we need to work with more flexible tools and commands, in particular, we need to obtain in realtime the orbit of a set of spherical points under the action of a (sub)group of spherical isometries. For that, GeoGebra [6] seems

${ }^{*}$ DOI: https://oi.org/10.1007/s12045-019-0849-6

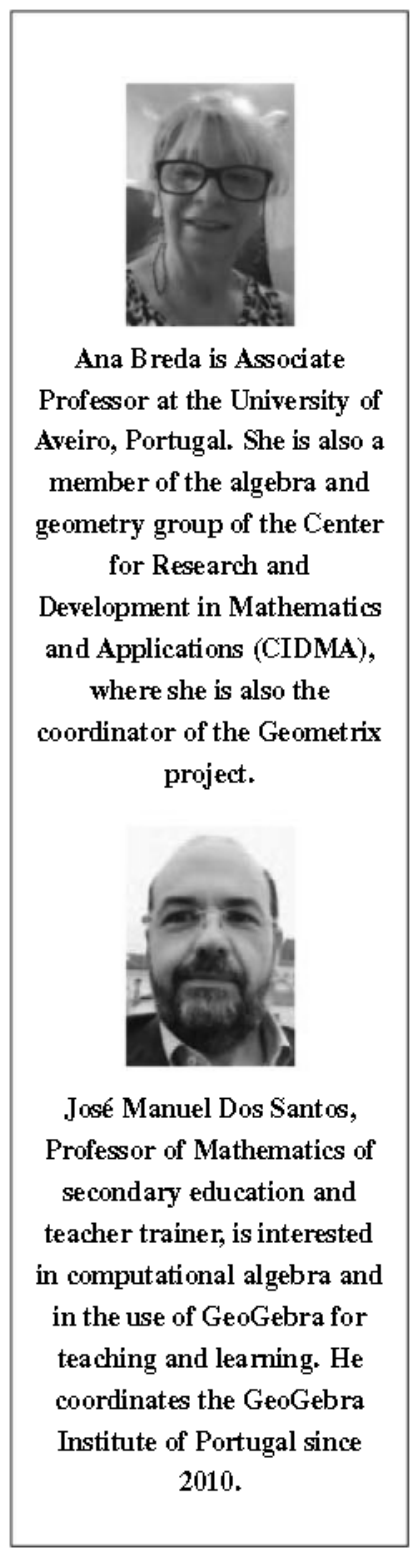

Professor of Mathematics of secondary education and teacher trainer, is interested computational algebra an teaching and learning. $\mathrm{He}$ coordinates the GeoGebra 2010. 


\section{Keywords}

GeoGebra, tiling, spherical geometry, polygon, points, straight lines, isometry. to be the best option for two crucial reasons: the widespread use of GeoGebra and the possibility of interaction with geometrical and algebraic representations simultaneously. GeoGebra has several geometrical representations in two and three dimensions allowing the interaction with spherical points in a diversity of ways. Besides, the algebraic capabilities of GeoGebra allow the study and induction of some geometrical properties which may be visualized in real-time. Among its many features, GeoGebra allows the creation of new tools and commands, dealing with sequences of various geometric and algebraic objects and using logical and heuristic procedures, permitting the certification of some properties of these same objects, as for example, to be congruent among them.

Our goal is, firstly, to use GeoGebra for the generation and visualisation of any regular triangular spherical tiling, followed by the generation and visualisation of monohedral spherical tilings whose prototile cell is a polygon, not necessarily triangular or even convex.

Within this goal, we have created new GeoGebra tools for spherical geometry.

\section{Octahedral Spherical Tiling with GeoGebra and Spatial Geometric Transformations}

Using parametrizations, we may colour the eight octants corresponding to the eight spherical triangles that constitute the monohedral octahedral tilling of the sphere, (see Figure 1a). One possibility is making use of the lateral surface command (see Figure $1 \mathrm{~b}$ ), using this command eight times and coloring the spherical triangles with different colours.

Using spherical isometries, we may also construct an application to get what is illustrated in Figure 1. Using two different colors for two adjacent spherical triangles, we obtain the other 6 by rotations and rotor-reflections of these two spherical triangles, (see Figure 2a). 
GENERAL ARTICLE

\section{(a) Application view}

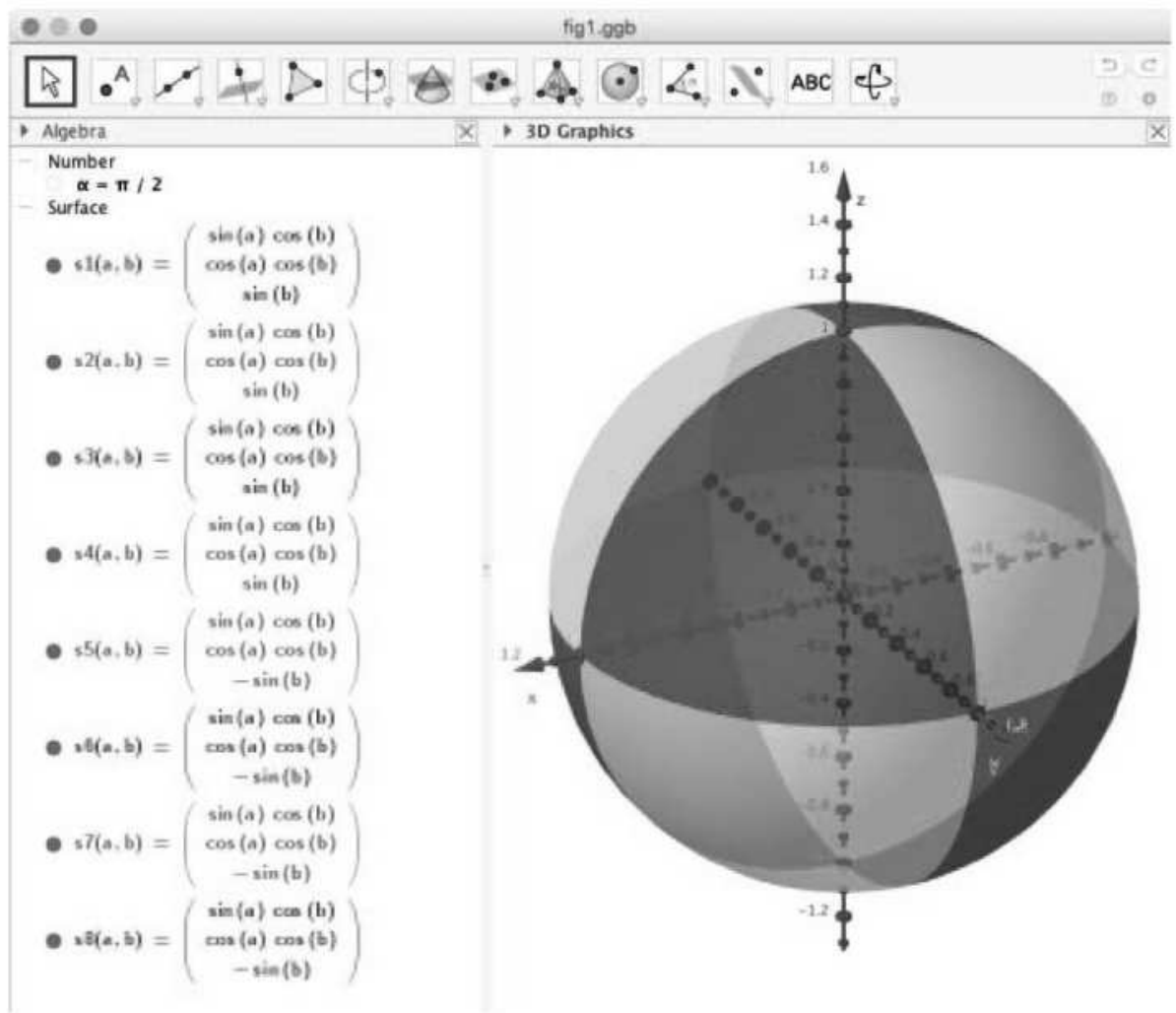

\section{(b) Commands}

$\alpha=\frac{\pi}{2}$

$\mathrm{sl}=$ Surface (sin(a) $\cos (\mathrm{b}), \cos (\mathrm{a}) \cos (\mathrm{b}), \sin (\mathrm{b}), \mathrm{a}, 0, \alpha, \mathrm{b}, 0, \alpha)$

$\mathrm{s} 2=$ Surface (sin(a) $\cos (\mathrm{b}), \cos (\mathrm{a}) \cos (\mathrm{b}), \sin (\mathrm{b}), \mathrm{a}, \pi+\alpha, \pi+2 \alpha, \mathrm{b}, 0, \alpha)$

$s 3=$ Surface (sin(a) $\cos (\mathrm{b}), \cos (\mathrm{a}) \cos (\mathrm{b}), \sin (\mathrm{b}), \mathrm{a}, \pi, \pi+\alpha, \mathrm{b}, 0, \alpha)$

s4=Surface( $\sin (\mathrm{a}) \cos (\mathrm{b}), \cos (\mathrm{a}) \cos (\mathrm{b}), \sin (\mathrm{b}), \mathrm{a}, \alpha, \pi, \mathrm{b}, 0, \alpha)$

$\mathrm{s} 5=$ Surface $(\sin (\mathrm{a}) \cos (\mathrm{b}), \cos (\mathrm{a}) \cos (\mathrm{b}),-\sin (\mathrm{b}), \mathrm{a}, 0, \alpha, \mathrm{b}, 0, \alpha)$

s6=Surface( $\sin (\mathrm{a}) \cos (\mathrm{b}), \cos (\mathrm{a}) \cos (\mathrm{b}),-\sin (\mathrm{b}), \mathrm{a}, \pi+\alpha, 2 \pi, \mathrm{b}, 0, \alpha)$

$s 7=$ Surface $(\sin (\mathrm{a}) \cos (\mathrm{b}), \cos (\mathrm{a}) \cos (\mathrm{b}),-\sin (\mathrm{b}), \mathrm{a}, \pi, \pi+\alpha, \mathrm{b}, 0, \alpha)$

$\mathrm{s} 8=$ Surface $(\sin (\mathrm{a}) \cos (\mathrm{b}), \cos (\mathrm{a}) \cos (\mathrm{b}),-\sin (\mathrm{b}), \mathrm{a}, \alpha, \pi, \mathrm{b}, 0, \alpha)$

Figure 1. Octahedral spherical tiling using parameterizations in GeoGebra. 


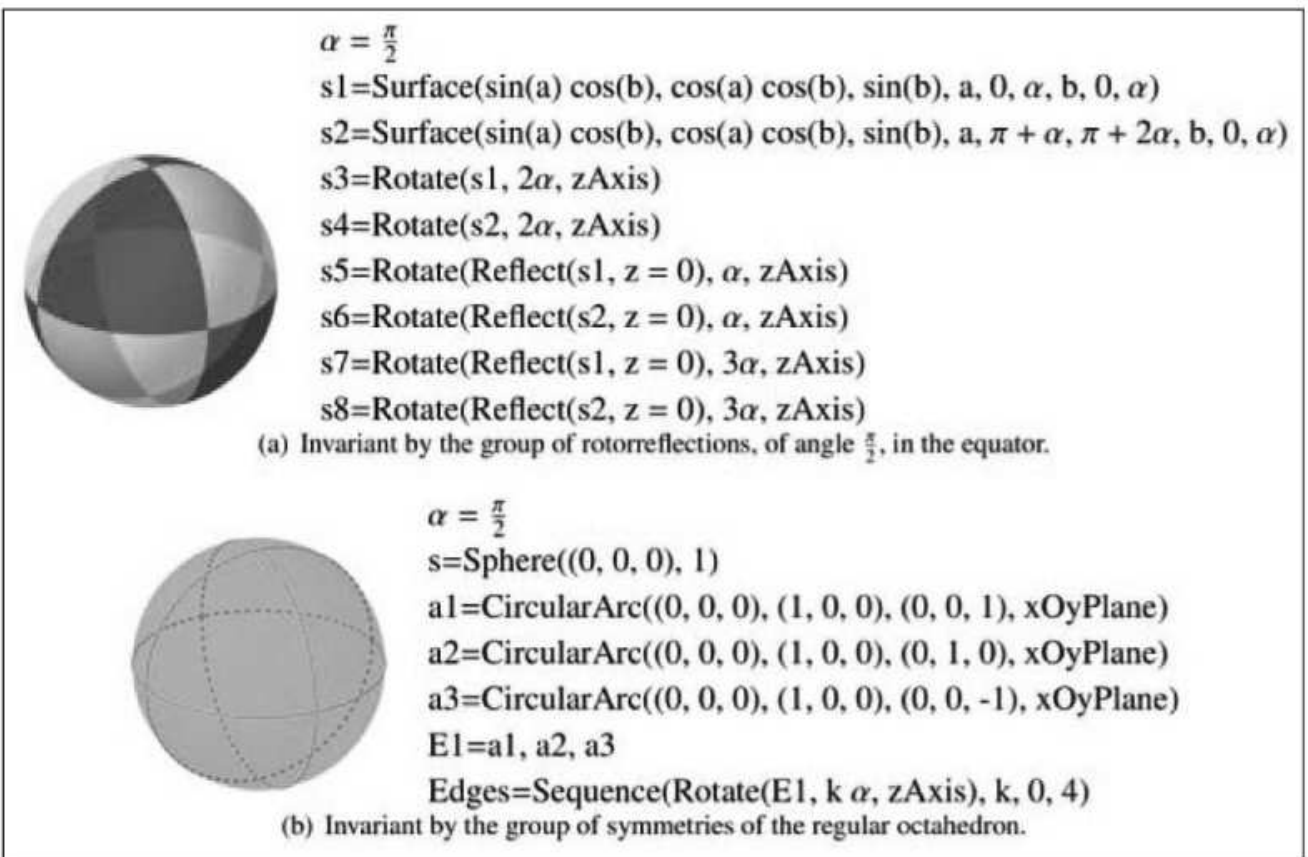

Figure 2. Octahedral spherical tiling obtained by different ways of construction of GeoGebra.

We assume, without loss of generality a sphere of radius 1 centered at the origin.
In Figure 2b, we see three great circles intersecting at right angles, dividing the sphere into eight congruent, equilateral and rightangled spherical triangles.

\section{New Tools in GeoGebra for Spherical Geometry}

In spherical geometry, the primitive elements 'point and 'straight lines' are modelled, respectively, by points in the sphere, $S^{2}=$ $\left\{(x, y, z) \in \mathbf{R}^{3}: x^{2}+y^{2}+z^{2}=1\right\}$, and great circles obtained by the intersection of $S^{2}$ with planes passing through the centre of $S^{2}$.

In GeoGebra, spherical points can be obtained making use of the point tool and commands $A=$ Point [s].

Let $A$ and $B$ be two distinct spherical non antipodal points. Using the command $s=$ Sphere $[(0,0,0), 1], s$, there is one and only great 


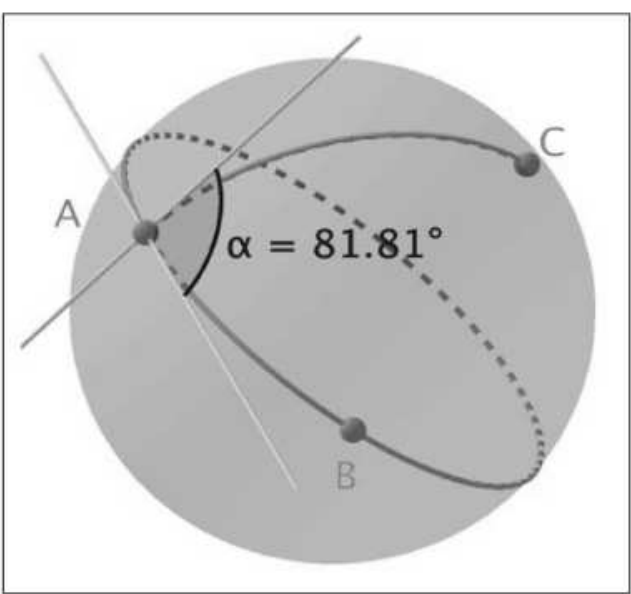

circle, $r$, containing $A$ and $B$. In GeoGebra, the representation of the line $A B$ will be given by:

$\mathrm{r}=\mathrm{Circle}(\mathrm{Centre}(\mathrm{s}), \mathrm{A}, \mathrm{Plane}(\mathrm{Centre}(\mathrm{s}, \mathrm{A}, \mathrm{B}))$

In accordance, the representation of the spherical segment $A B$ would be:

$\mathrm{AB}=$ CircularArc (Centre ( $\mathrm{s}), \mathrm{A}, \mathrm{B}$, Plane

(Centre (s), A,B))

(Centre (s), A,B ))

A spherical polygon (concave or convex) corresponds to a spherical region bounded by spherical segments.

Another important element in spherical geometry is the angle defined by two spherical segments. Given three points $A, B$ and $C$ on the sphere the angle $B A C$ corresponds to the angle defined by the tangent lines to the spherical segments $A B$ and $A C$ at the vertex A.

For the example illustrated in Figure 3, the angle $\alpha$ was defined using the command:

Angle (Tangent (A, CircularArc (Centre (s), A,C, Plane (Centre (s), A,C))), Tangent (A, Circle (Centre (s), A, Plane (Centre (s), A,B ))) )
Figure 3. Points, straight lines, straight line segments, and angles in spherical geometry.
A spherical polygon (concave or convex) corresponds to a spherical region bounded by spherical segments. 


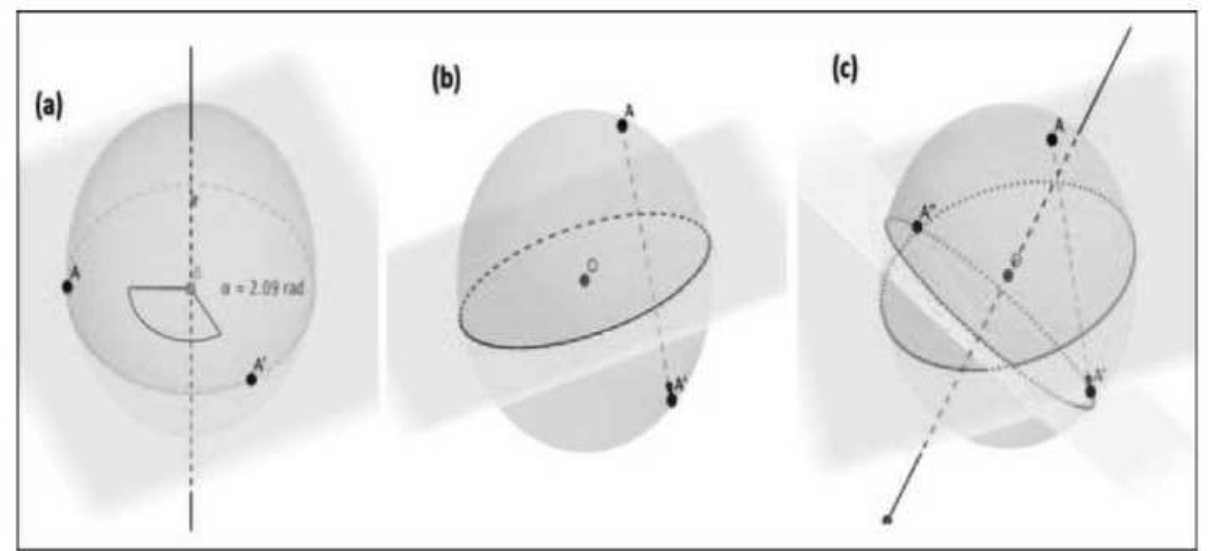

Figure 4. Isometries of the sphere (a) Rotation (b) Reflection (c) Rotor-reflection.
Following this logic of object construction, we have created tools in GeoGebra, allowing the real-time construction of spherical segments and distances between points and angle measures, allowing the construction of different spherical configurations with control in angle and distance point measure enabling us a great deal of flexibility for the exploration of spherical patterns.

\section{Spherical Isometries}

As it is well known, the spherical isometries - transformations of the sphere preserving the spherical distance - are rotations about an axis passing through their center (Figure 4a); plane reflections passing through its center (Figure $4 \mathrm{~b}$ ), compositions of a reflection in a plane passing through its center followed by a rotation about an axis perpendicular to this plane passing through the center (Figure 4c) and any composition of the isometries already mentioned.

The image $A^{\prime}, B^{\prime}, C^{\prime}$ of 3 spherical points, $A, B, C$ not belonging to the same large circle univocally determine a spherical isometry, $f$, satisfying, $f(A)=A^{\prime}, f(B)=B^{\prime}, f(C)=C^{\prime}$.

In Figure 5, it can be visualised the composition of two reflections in planes passing through the center of the sphere, the rotation about the axis obdefined by the intersection of the two planes of reflection. 


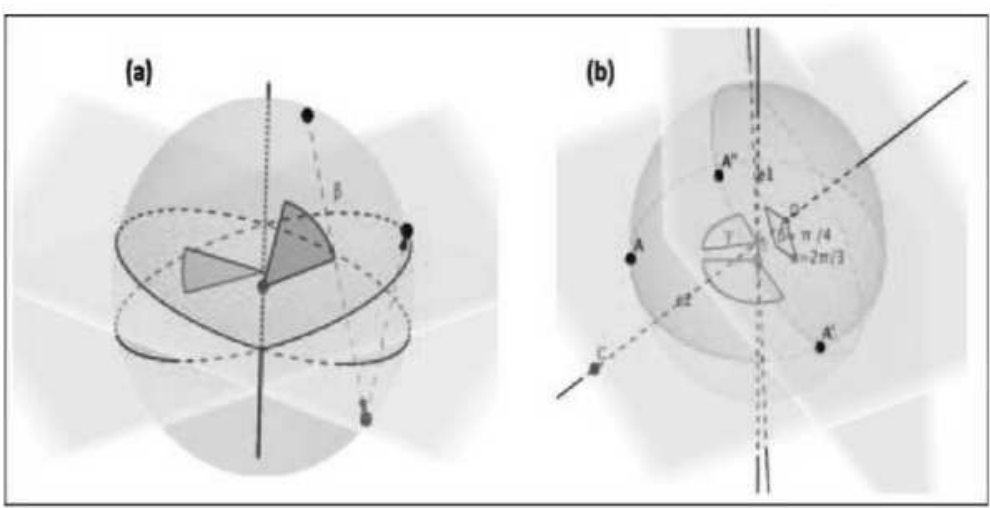

5. Locus in Spherical Geometry with Spherical Compass Tool

In Euclidean geometry, the constructions with ruler and compass play an important role. With the spherical compass GeoGebra tool, we can explore similar constructions in spherical geometry.

One of the simplest constructions in Euclidean geometry corresponds to the perpendicular bisector of a 'straight line segment'. In the sphere of center $O$ and radius $r$, we may use the spherical compass tool to perform the same type of construction.

Considering two spherical points $A$ and $B$ and a distance $l$ (controlled by a selector) and defining $P$ by:

$\mathrm{P}=$ Intercept (SphereCompass $(\mathrm{A}, 1, \mathrm{O}, \mathrm{r})$,

SphereCompass $(\mathrm{B}, \mathrm{l}, \mathrm{O}, \mathrm{r}))$.

$P$ corresponds to the set of all spherical points equidistant from $A$ and $B$, which is precisely the large circle perpendicular to the spherical segment $A B$ (see Figure 6 a) passing through the midpoint of $A B$.

Similarly, given two points $A$ and $B$ on the sphere, and a distance $l$, we can use the spherical compass tool to construct the set of points $P$, on the sphere, such that $d_{\varepsilon}(P, A)+d_{\varepsilon}(P, B)=l$. This
Figure 5. Composition of two reflections in $S^{2}$.
GeoGebra can also help to obtain the locus equation using the $C A S$ View. 
Figure 6. Locus on the Sphere (a) Mediatrix of a spherical segment (b) Spherical ellipse (c) Spherical Parabola.

Using the Spherical Compass and starting from a net of $n$ congruent equilateral triangles, depending on the initial points $A$ and $B$, and moving these points around the sphere, we can explore many configurations being some of them spherical tilings.

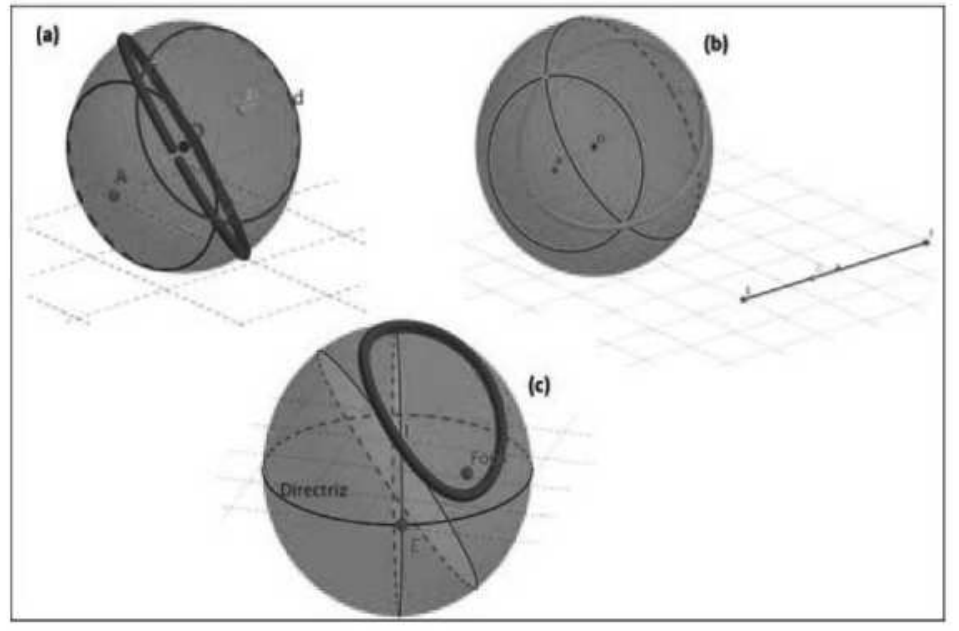

set of points corresponds to the 'spherical ellipse' shown in Figure $6 \mathrm{~b}$. The notions of straight lines and parabolas (unrestricted curves in the open set $\mathbf{R}^{2}$ ), and making use of the spherical compass tool it is easy to visualize the equivalent spherical notions, which correspond to the closed curves illustrated in Figure $6 \mathrm{c}$.

\section{From the $S T e q A B[A, B, O, r]$ Tool to the Regular Spherical Tilings}

Associated to the constructed spherical tool, Spherical Compass is the command $S T e q A B[A, B, O, r]$ used to construct equilateral spherical triangles.

Using the Spherical Compass and starting from a net of $n$ congruent equilateral triangles, depending on the initial points $A$ and $B$, and moving these points around the sphere, we can explore many configurations being some of them spherical tilings.

In Figure 7 we illustrate this procedure, using nets with three, eight and twenty triangles ending up in the tetrahedral, octahedral and icosahedral regular spherical tilings. Using the same strategy with another net of triangles, for example, with common vertices or adjacent sides, and observing the evolution of the set according to the different positions of the initial points, we may explore the 
GENERAL ARTICLE

\begin{tabular}{|c|c|c|c|c|}
\hline $\begin{array}{l}\text { Using a set of } n \\
\text { equilateral } \\
\text { triangles }\end{array}$ & ....in one hemisphere & $\begin{array}{l}\text { Length of segment } \\
A B\end{array}$ & ....closing around one vertex & $\begin{array}{l}\text { Final length of segment } \\
A B\end{array}$ \\
\hline 3 & & $1.05 \mathrm{rad}$ & & $\begin{array}{l}\arccos \left(-\frac{1}{3}\right) \\
=2 \arctan (\sqrt{2}) \\
=1.908 \mathrm{rad}\end{array}$ \\
\hline 8 & & $0.64 \mathrm{rad}$ & & $\frac{\pi}{2} \approx 1.574 \mathrm{rad}$ \\
\hline 20 & & $0.40 \mathrm{rad}$ & & $\begin{array}{l}2 \arcsin \left(\frac{\sqrt{5-\sqrt{5}}}{\sqrt{10}}\right) \text { o } \\
1.107 \mathrm{rad}\end{array}$ \\
\hline
\end{tabular}

possibilities to obtain new spherical tilings, see Figure 7.

Figure 7. Evolution of nets of equilateral and congruent

\section{Spherical Tiling as Global or Local Action of Groups of \\ Spherical Symmetries} triangles.

In the previous sections, we show how we can obtain regular tiling of the sphere (see Figures 2b and Figure 7). These are related to regular polyhedrons and their symmetry groups. In these special cases, starting from a spherical triangle, its orbit under the global action of a group of symmetries determines the spherical tiling.

Let us see another example obtained similarly.

Consider:

i) An axis, $e$, of the sphere $S$; ii) A point $A_{1}$, such that $A_{1} \in S$ and $A_{1} \notin e$; iii) Choose one of the points $P$, such that $P \in e \wedge S$; iv) The angle $\alpha=\frac{2 \pi}{n}, n \in \mathbf{N} \wedge n>3$; v) Let $\left(A_{n}\right)_{n \in \mathbf{N}}$ is the orbit of the point $A_{1}$ obtained by the action of the group of rotations of the sphere of angle $\alpha$ around the axis $e$.

Under these conditions, we obtain a tiling of the sphere consist- 
GENERAL ARTICLE

Figure 8. Application of GeoGebra to obtain a tiling of the sphere, invariant by a cyclic group of order equal to the value of selector $n$.

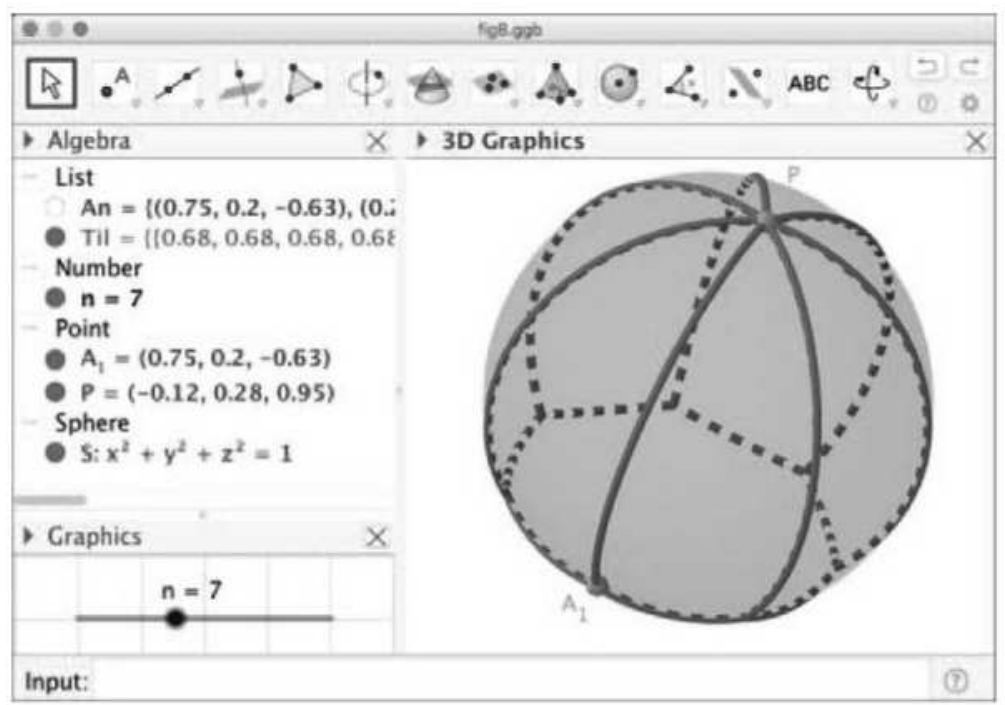

ing of a spherical $n$ - gono and $n$ congruent spherical triangles whose prototype is $\left[A_{1} A_{2} P\right]$. This tiling is generated by the cyclic group of order $n$. In the case of Figure 8, we have as a group of symmetries the cyclic group of order 7 , being the spherical tiling constituted by eight spherical polygons, one heptagon and seven triangles, this tiling is associated to a straight heptagonal pyramid.

The basic idea behind the construction of this type of tiling is to use the sequence command to model the point orbits. To do that, we use a sequence of commands with a syntax similar to:

$A n=$ Sequence $\left(\right.$ Rotate $\left(A_{-} 1,2 \$ \backslash \mathrm{pi} \$ \mathrm{i} / \mathrm{n}\right.$, Line (Centre (S),P)), i , 0, n, 1)

On the other hand, to obtain the spherical segments, which are the sides of the tiling polygons, we use the list:

Til $=\{$ Sequence $($ CircularArc $($ Centre $(S)$, Element (An, i ), Element (An, i + 1)), i , 1, n, 1), Sequence (CircularArc (Centre (S), Element $(A n, k), P), k, 1, n, 1)\}$

Many other spherical tilings can be 'found' by the local action analysis of (sub)spherical isometry groups. These tilings are less well known, and many are still to be studied. 


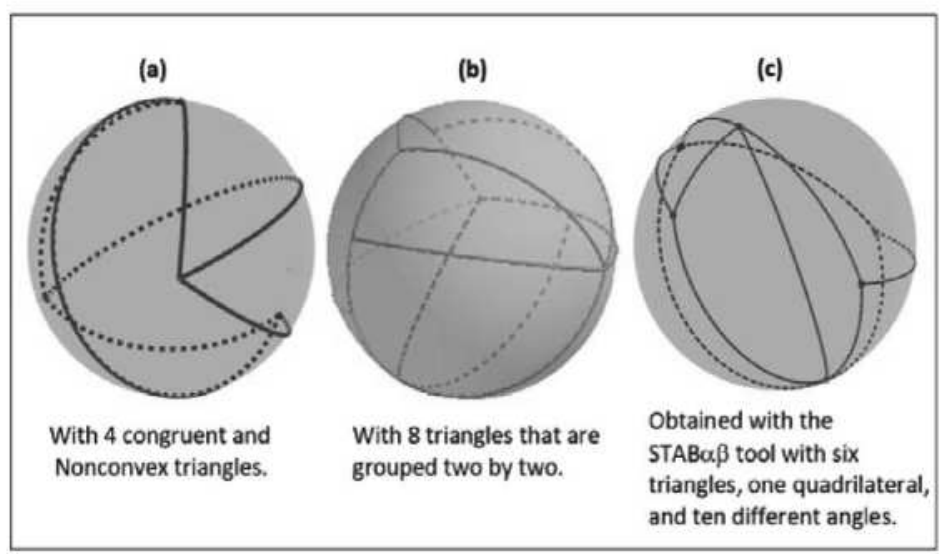

The tilings by non-convex spherical polygons (Figure 9a), especially the monohedral ones, is one of the cases that has not yet been studied so far. Also, the tilings that can integrate more than one type of spherical polygon (not necessarily regular (Figure 9c) and not necessarily convex), is another case that has not yet been explored.

In our recent work, using these tools, and from the iteration of a set of points $\mathscr{C}$ from a set of spherical isometries, we found several classes of monohedral spherical tilings. In Figure 10 we can see some of them: $\mathfrak{I}_{(\mathscr{L}, \rho)}$, (Figure 10a), composed of four congruent triangles of area $\pi ; \mathfrak{P}_{(\mathscr{L}, \rho)}$, (Figure 10b), composed of four spherical pentagons of area $\pi$; two elementes of $\mathfrak{S}(\mathscr{C}, \rho)$, in Figure 10c the tiling has six spherical hexagons, however in Figure 10(d) the tiling had six spherical pentagons, in both cases each tile have of area $\frac{2 \pi}{3}$.

The tiles of $\mathfrak{T}_{(\mathscr{E}, \rho)}$, for $\rho>\frac{\pi}{2}$ are not convex spherical polygons. The convex case was already described by several other authors, see for instance Brooks and Strantzen [7]. However, the nonconvex case, $\left.\mathfrak{I}_{(\mathscr{G}, \rho)}, \rho \in\right] \frac{\pi}{2}, \pi[$ as far as we know, is not mentioned in the literature. We only find a brief reference to $\mathfrak{I}_{(\mathscr{L}}$, arccos $\left.(-1 / 3)\right)$, in Figure 9a, by Gaiane in $[8,9]$. As far as we know, the class of monohedral spherical tiling, $\mathfrak{P}_{(\mathscr{b}, \rho)}$, by four spherical pentagons of area $\pi, \mathfrak{P}_{(\mathscr{L}, \rho)}$ is a new one [10].
Figure 9. Other spherical tilings.

The tilings by non-convex spherical polygons (Figure 9a), especially the monohedral ones, is one of the cases that has not yet been studied so far. 
Figure 10. Monohedral spherical tilings obtained iterating $\mathscr{C}$ under a set of local actions.
The GeoGebra applications built so far, allow the visualization and establishment of relationships that can greatly contribute to research in this area.

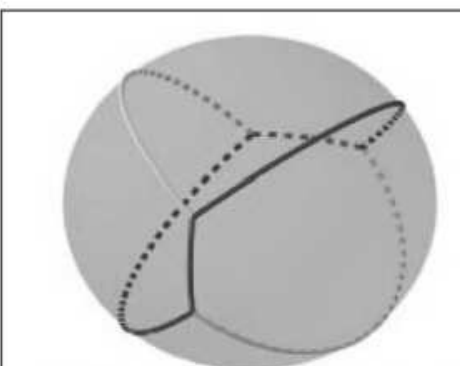

(a) Monohedral triangular spherical tiling. $\mathcal{Z}_{\left(C, \frac{\pi}{10}\right)}$.

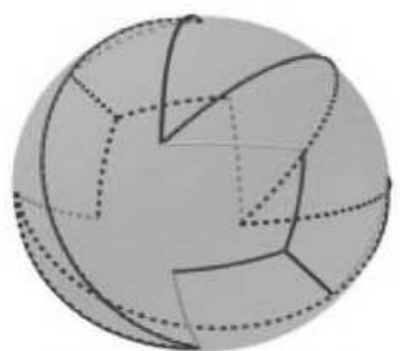

(c) Monohedral hexagonal spherical tiling, $S_{\left(C, \frac{7 n}{6}\right)}$.

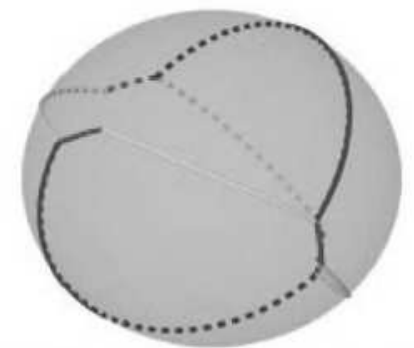

(b) Monohedral pentagonal spherical tiling, $\mathfrak{\vartheta}_{\left(C, \frac{\pi}{\pi}\right)}$.

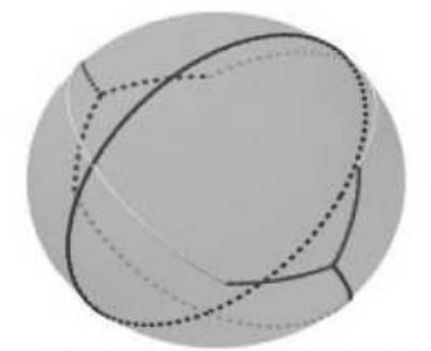

(d) Monohedral hexagonal spherical tiling, $\mathfrak{S}_{(C, 0)}$

\section{Conclusion}

The GeoGebra applications built so far, allow the visualization and establishment of relationships that can greatly contribute to research in this area. It is in the generation of such a great variety of spherical configurations/relationships that we believe GeoGebra can make a substantial contribution to the description and construction of spherical tilings not yet explored, besides being a resource of great utility in the study of spherical geometry, in general.

\section{Acknowledgement}

This work was supported in part by the Portuguese Foundation for Science and Technology (FCT - Fundacäo para a Ciência e a Tecnologia), through CIDMA - Centre for Research and Development in Mathematics and Applications, within project UID/MAT/04106/2019. 


\section{Suggested Reading}

[1] DMY Sommerville. VI.-Division of Space by Congruent Triangles and Tetrahedra, Proceedings of the Royal Society of Edinburgh, 43, pp.85-116, 1924.

[2] Yukako Ueno and Yoshio Agaoka, Tilings of the 2-dimensional Sphere by Congruent Right Triangles, Memoirs of the Faculty of Integrated Arts and Sciences, Hiroshima University, IV, Science reports: Studies of Fundamental and Environmental Sciences, 22, 1996. Available online at http: //ir.lib.hiroshimau.ac.jp/files/public/0/673/20141016120323365034/KJ00000197303 .pdf, pp.123.

[3] János Erdõs, Sphaerica: Interactive Spherical Geometry Software, Proceedings of Bridges 2010: Mathematics, Music, Art, Architecture, Cul ture, Ed. by George W Hart and Reza Sarhangi. Available online at http://archive.bridgesmathart.org/2010/bridges2010-443 . html. Phoenix, Arizona: Tessellations Publishing, 2010, pp.443-446. isbn: 978-0-98460-420-3.

[4] D Austin and W Díckinson, Spherical Easel, A Spherical Drawing Program. Available online at http://mergansermath.gvsu.edu/easel/. 2009.

[5] Robert J MacG Dawson et al., Some New Tilings of the Sphere with Congruent Triangles, Renaissance Ban: Mathematics, Music, Art, Culture. Bridges Conference, pp.489-496, 2005.

[6] Markus Hohenwarter and Karl Fuchs. Combination of Dynamic Geometry, Algebra and Calculus in the Software System GeoGebra, Computer Algebra Systems and Dynamic Geometry Systems in Mathematics Teaching Conference, Available online at http:/archive.geogebra. org/static/publications/pes__2004.pdf. 2004.

[7] Je Brooks and John Strantzen, Spherical Triangles of Area and Isosceles Tetrahedra, Mathematics Magazine, 78.4, pp.311-314, 2005.

[8] Gaiane Panina, Planar Pseudo-triangulations, Spherical Pseudotilings and Hyperbolic Virtual Polytopes, arXiv preprint math/0607171, 2006.

[9] G Yu Panina, Pointed Spherical Tilings and Hyperbolic Virtual Polytopes, Journal of Mathematical Sciences, 175.5, pp.591-599, 2011; G Yu Panina, Pointed Spherical Tilings and Hyperbolic Virtual Nolytopes, Jotonal of Mathematical Sciences, 175.5, 2011, pp.591-599.

[10] Ana Maria d'Azevedo Breda and José Manuel Dos Santos Dos Santos, 'A New Class of Monohedral Pentagonal Spherical Tilings with GeoGebra', Portugaliae Math, 74.3, In press, pp.257-266, 2018.
Address for Correspondence Ana Breda

University of Aveiro

Campus Universitário de

Santiago

3810-193 Aveiro Portugal

Email: ambreda@ua.pt

José Dos Santos

Portuguese Institute of

$$
\text { GeoGebra }
$$

Email: dossantosdossantos agmail.com 